\title{
El nivel de coherencia, cohesión y ortografía de los profesores de Educación Básica Regular de Lima Metropolitana
}

\section{The level of coherence, cohesion and spelling of Basic Education Teachers of Lima}

\author{
Miguel Gerardo Inga Arias, Pedro Rojas Silva y Fernando Varas Aroné \\ Universidad Nacional Mayor de San Marcos
}

DOI: https://doi.org/10.33017/RevECIPeru2015.0015/

\section{Resumen}

Históricamente se ha considerado que el problema de la producción de textos tenía que ver, sobre todo, con la correcta escritura, limitándola a la buena ortografía o caligrafía. A partir de la lingüística textual, esta concepción ha sido superada, pues producir un texto implica, en primer lugar, un propósito, una intención, un qué reacción verbal o extraverbal espero lograr; para ello se recogen palabras, conceptos y toda información considerada importante para lograrlo. En segundo lugar, se produce la textualización, la cual consiste en relacionar ideas, que deben tener secuencia lógica, así como cantidad de información necesaria y suficiente que permita la propiedad, claridad y pertinencia del discurso, esto es, coherencia. Además, ensamblar adecuadamente las frases y construcciones lingüísticas para alcanzar la fluidez y evitar la redundancia, es decir, cohesión. También, debemos tener muy en cuenta al interlocutor, ya que todo acto comunicativo debe garantizar el nivel de código que sea accesible a las partes, en otras palabras, adecuación. En tercer lugar, recién se procede a la revisión formal del texto, tanto de los recursos lingüísticos como de los paralingüísticos: la ortografía, que es el revestimiento de las ideas; y espacios, márgenes, tipo de letras, entre otros. En consecuencia, esta investigación buscaba diagnosticar el nivel de dominio de la coherencia, cohesión y ortografía de los profesores de la Educación Básica Regular (EBR) para, posteriormente, proponer estrategias que les garanticen los niveles adecuados. Además, permitiría la capacitación focalizada en los puntos neurálgicos, con lo cual se racionalizarían tanto el tiempo como recursos económicos. La información se logró a través de la aplicación de instrumentos de evaluación a los participantes de las maestrías del primer ciclo de la Facultad de Educación, por ser una muestra muy representativa en la docencia de la EBR.

Descriptores: Coherencia, cohesión, ortografía, comprensión lectora

\begin{abstract}
Historically it was thought that the problem of the production of texts had to do, especially with the correct spelling, limiting it to the correct spelling or handwriting. From textual linguistics, this conception has been overcome, it produce a text implies , first, a purpose, an intention, a verbal reaction or extraverbal what I hope to accomplish, for it is collected words, concepts and all information deemed important to achieve it. Second, contextualization occurs, which is to relate ideas, which should be logical and amount of information necessary and sufficient to enable the property, clarity and relevance of discourse, ie consistency. Furthermore, phrases and properly assemble linguistic constructs to achieve fluidity and avoid redundancy, ie cohesion. Also, we must take into account the speaker, as any communicative act should ensure code level that is accessible to the parties, in other words, adaptation. Third, just proceed to the formal review of the text, both the linguistic and the pralinguistic: spelling, which is the lining of ideas and spaces, margins, fonts, and more. Consequently, this research sought to assess the level of mastery of coherence, cohesion and spelling teachers Basic Education (EBR) to subsequently propose strategies that will ensure adequate level. Also allow training
\end{abstract}


focused on the key points, which is both time and rationalize financial resources. The information is achieved through the application of assessment tools to participants of the masters of the first cycle of the Faculty of Education, being a very representative sample in the teaching of the EBR.

\section{Keywords: Coherence, cohesion, spelling, comprehension reading}

\section{Introducción}

Nuestra preocupación respecto al problema de la lectura es una constante en nuestro trabajo de investigación. En esta oportunidad hemos empezado el estudio de la relación con los microprocesos de la escritura, particularmente con la redacción. Si la lectura es procesar información de los textos, estos no pueden ser la copia de otro texto; en consecuencia, producir textos es establecer relaciones de ideas con un propósito [1]. Si los profesores no tienen claridad en ello, tendrán serios problemas con la comprensión y producción de textos, es decir, con el procesamiento de información y, por consiguiente, también sus alumnos.

Por ello, la hipótesis de trabajo tiene que ver con identificar el nivel de dominio de la coherencia, cohesión y ortografía de los profesores de la Educación Básica Regular (EBR), pues estos factores son fundamentales para explicar sus problemas de comprensión lectora, los cuales influyen significativamente en las limitaciones de comprensión lectora en sus alumnos.

Esta investigación permitió identificar las habilidades de organizar y relacionar ideas (coherencia), claridad y precisión de cláusulas y párrafos (cohesión) y de recursos en el uso de grafías, tilde, puntuación, mayúsculas para precisar significados (ortografía) de los profesores de EBR [2].

\section{Diseño experimental o metodológico}

El diseño, por ser una investigación de carácter descriptivo, consistió, primero, en definir, dentro del Equipo de trabajo, los procesos mentales de la comprensión lectora y de la producción de textos, así como de su interacción. En segundo lugar, definir la tipología textual pertinente, es decir, la decisión tenía que estar en función de la información más adecuada para medir la coherencia, cohesión y ortografía. En tercer lugar, el formato del instrumento, el cual fue elaborado sobre la base de instrumentos validados en las cátedras universitarias en nuestra universidad. Cabe resaltar su nivel de confiabilidad, pues al haberse aplicado a promociones distintas, los resultados en grupos medianamente homogéneos fueron muy similares.
Cada una de las pruebas fue aplicada en un tiempo determinado. La de coherencia y cohesión en un solo momento. La de ortografía en otro momento. La condición de ser profesores en ejercicio en la EBR de distintas instituciones educativas de Lima Metropolitana, haber pasado por un proceso de selección para ingresar a estudiar la Maestría en la Facultad de Educación de la Universidad Nacional Mayor de San Marcos, hace que sean una muestra altamente significativa y representativa de la docencia de la Educación Básica Regular.

Las pruebas aplicadas estuvieron enmarcadas como parte de la evaluación del área académica de Comunicación con lo cual se garantizaba la seriedad por resolver los instrumentos que preparáramos especialmente para investigación. Por consiguiente, logramos controlar cualquier factor interviniente que podría haber distorsionado la aplicación de estas pruebas.

\section{Muestra poblacional}

Todos los maestristas ingresantes de la mención Didáctica de la Comunicación en Educación Básica Regular del año 2013.

Optamos por aplicar esta investigación en a los maestristas de esta mención por las siguientes razones:

Los maestristas han pasado por un proceso de selección que permite haber seleccionado participantes con mejor nivel y, sobre todo, están un tanto más homogeneizados respecto a otros grupos.

Los participantes son de diferentes niveles de Educación Básica Regular, pero todos relacionados con la especialidad de Lengua y Literatura, lo que hace que se constituya como un grupo con información más cercana que otros grupos.

La composición económicosocial de los participantes es bastante similar.

El grupo participa de la misma modalidad denominada bimodal, pues las clases son presenciales y con apoyo virtual, sin embargo, los materiales de apoyo son los mismos. 
La composición del grupo presentaba una presencia similar entre varones y mujeres, respecto a las edades oscilaban como promedio los 35 años de edad.

Estos grupos presentan regularidad de su presencia en clases de los fines de semana, lo cual nos permitió observar que respecto a estos factores de investigación, no tenían otra capacitación, que pudiera distorsionar los resultados.

\section{Procedimiento}

Una semana después se les aplicó la prueba de Ortografía. El tiempo fue de 40 minutos. El tiempo que se les otorgó estuvo calculado en función de los ítems propuestos.

Sabemos que el dominio tiene que ver con el manejo de la información, el cual pasa por una serie de procesos mentales como el de identificar, seleccionar, organizar y redactar, mas, también, es un aspecto a tomar en cuenta el tiempo. Ello permite disminuir el factor azaroso que está presente como posibilidad en instrumentos sin puntaje en contra, que se suele dar cuando las respuestas son inadecuadas.

Tabla 1: Resultados de la prueba de Ortografía según tipo ítems.

\begin{tabular}{|l|r|r|r|r|}
\hline Estadístico & \multicolumn{1}{|c|}{$\begin{array}{c}\text { Uso de } \\
\text { mayúsculas }\end{array}$} & $\begin{array}{c}\text { Palabras } \\
\text { juntas o } \\
\text { separadas }\end{array}$ & $\begin{array}{c}\text { Grafías } \\
\text { faltantes y } \\
\text { tildes }\end{array}$ & $\begin{array}{c}\text { Signos de } \\
\text { puntuación }\end{array}$ \\
\hline N Válidos & 46 & 46 & 46 & 46 \\
\hline Media & 13,00 & 18,70 & 12,70 & 16,544 \\
\hline Mediana & 16,00 & 20,00 & 13,00 & 16,800 \\
\hline Moda & 18 & 20 & 12 & 16,8 \\
\hline Desv. típ. & 5,379 & 2,117 & 2,772 & 2,3040 \\
\hline Varianza & 28,933 & 4,483 & 7,683 & 5,308 \\
\hline Mínimo & 4 & 12 & 4 & 7,6 \\
\hline Máximo & 20 & 20 & 17 & 19,6 \\
\hline Suma & 598 & 860 & 584 & 794,1 \\
\hline
\end{tabular}

Tabla 2: Resultados de la Prueba de Coherencia y de Cohesión

\begin{tabular}{|l|r|r|}
\hline Estadístico & \multicolumn{1}{|c|}{$\begin{array}{c}\text { Prueba de } \\
\text { cohesión }\end{array}$} & \multicolumn{1}{|c|}{$\begin{array}{l}\text { Prueba de } \\
\text { coherencia }\end{array}$} \\
\hline N Válidos & 48 & 48 \\
\hline Media & 12,88 & 12,552 \\
\hline Mediana & 13,00 & 12,500 \\
\hline Moda & 13 & 20,0 \\
\hline Desv. típ. & 4,216 & 6,1775 \\
\hline
\end{tabular}

\begin{tabular}{|l|r|r|}
\hline Varianza & 17,771 & 38,162 \\
\hline Mínimo & 1 &, 0 \\
\hline Máximo & 20 & 20,0 \\
\hline Suma & 618 & 602,5 \\
\hline
\end{tabular}

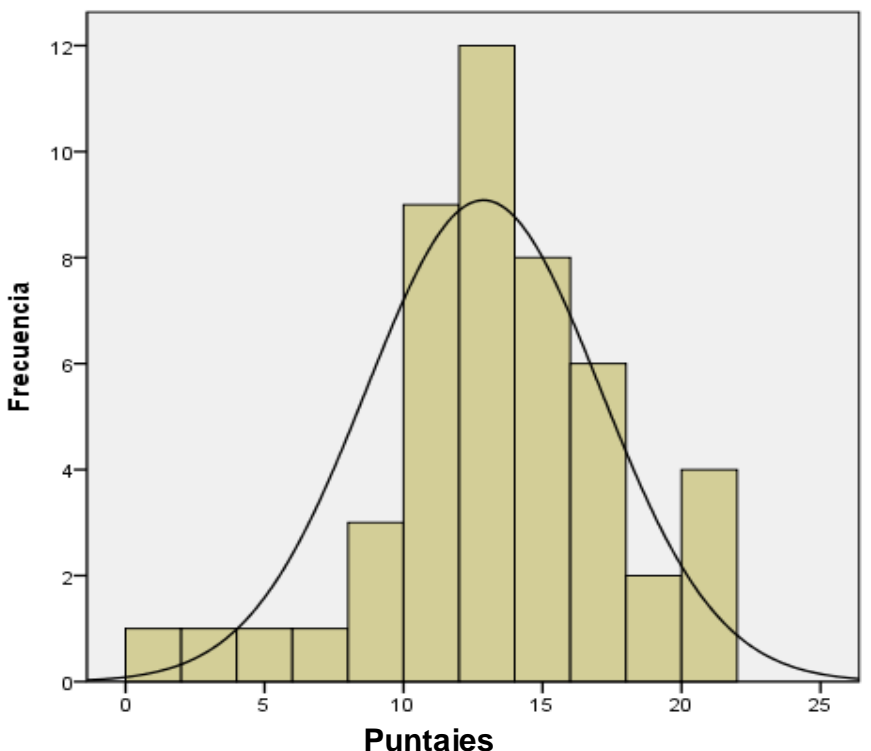

Figura 1: Frecuencia de los puntajes de la Prueba de cohesión.

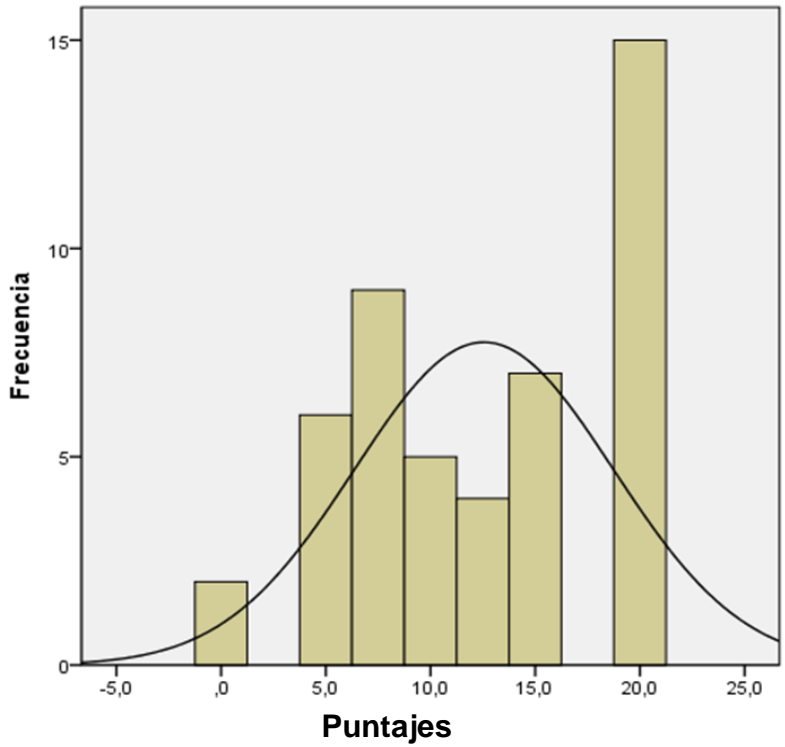

Figura 2: Frecuencia de los puntajes de la Prueba de coherencia.

\section{Resultados y discusión}

Esta investigación ha permitido tomar conciencia respecto a la íntima relación que se da entre la comprensión lectora y la producción de textos, como procesos interactuantes. Es por eso que no debemos desvincular sus actividades, pues conociendo el modo de cómo se elabora un texto el acercamiento al mismo es más asequible y, en su lectura, la reconstrucción de su sentido es más profunda y más ordenada. 
Cohesión, coherencia y ortografía son exigencias que todo texto debe cumplir para ser reconocido como tal. La claridad, propiedad y pertinencia significan herramientas necesarias para una mejor comprensión de la lectura. De ahí su íntima vinculación con la producción de textos, pues ambos constituyen el circuito de la comunicación plena [3].

Es preocupante que en docentes que han transcurrido cinco años de formación profesional en la especialidad de Lengua y Literatura o, en el caso de Primaria, con incidencia en la especialidad de Lenguaje, obtengan nota como promedio de 12,5 en la Prueba de Coherencia y, también, en la Prueba de Cohesión. Además, son profesores con un promedio de seis años de experiencia profesional.

Es más preocupante que en la Prueba de Ortografía hayan obtenido trece (13) como promedio en Uso de mayúsculas y 12,7 en Uso de tildes y Uso de grafías. Si observamos las pruebas, nos percataremos que los términos empleados en los ejercicios propuestos todos son de uso frecuente, así como contextualizados, pues están incursos en textos.

Notamos que en el caso de la subprueba de Palabras Juntas y Separadas el promedio es alto, 18,7, mas es esta tiene que ver más con la formalidad de la escritura que con la comprensión del texto. Esto evidenciaría la formación reglamentarista del docente antes que la reflexiva. Algo similar encontramos cuando vemos los resultados de la subprueba de Signos de Puntuación cuyo promedio es 16,54.

Esta afirmación se sustenta porque la subprueba de Signos de Puntuación presenta oraciones a las cuales les falta ubicar signos de puntuación de acuerdo a las reglas clásicas; en cambio, cuando los debemos emplear reflexivamente como mecanismos de cohesión vemos deficiencias en su uso, lo cual revela problemas de comprensión del texto.

Estos resultados revelan que el sistema educativo no solo entrampa el desarrollo cognitivo de los estudiantes; también de los docentes, constituyéndose un círculo vicioso. Los docentes que presentan estos problemas no podrán promover la comprensión lectora si ellos presentan estos problemas.

Definitivamente, es esencial que abordemos en las instituciones educativas como eje del desarrollo del área de Comunicación la comprensión lectora y la producción de textos como dos aspectos íntimamente relacionados, con estrategias de carácter cognitivo y metacognitivo para lo cual los textos escolares deben orientarse en ese sentido.

\section{Conclusiones}

Producir un texto implica, en primer lugar, un propósito, una intención, un qué reacción verbal o extraverbal espero lograr; para ello se recogen palabras, conceptos y toda información considerada importante para lograrlo. En segundo lugar, se produce la textualización, la cual consiste en relacionar ideas, que deben tener secuencia lógica, así como cantidad de información necesaria y suficiente que permita la propiedad, claridad y pertinencia del discurso, esto es, coherencia. Además, ensamblar adecuadamente las frases y construcciones lingüísticas para alcanzar la fluidez y evitar la redundancia, es decir, cohesión. También, debemos tener muy en cuenta al interlocutor, ya que todo acto comunicativo debe garantizar el nivel de código que sea accesible a las partes, en otras palabras, adecuación. En tercer lugar, recién se procede a la revisión formal del texto, tanto de los recursos lingüísticos como de los paralingüísticos: la ortografía, que es el revestimiento de las ideas; y espacios, márgenes, tipo de letras, entre otros.

La formación profesional de los docentes, tanto en universidades como en pedagógicos, debe centrarse en el desarrollo de sus capacidades lingüísticas y comunicativas, así como en la reflexión sobre ellas, para que puedan estar preparados para proponer y aplicar estrategias que permitan disfrutar y comprender diversos tipos de textos. Sin embargo, la formación centrada en información gramatical y de teoría lingüística no potencia las habilidades de lectura y comprensiva de los docentes.

Las capacidades de selección de ideas fundamentales, organización de la información y capacidad de síntesis, que son microhabilidades de la lectura comprensiva son muy limitadas en los docentes de Educación Básica, ello se hace evidente cuando presentan baja performance en las pruebas de Coherencia, Cohesión y, en el caso de la de Ortografía, particularmente en uso de mayúsculas, tildes y grafías.

Este problema de falta de perspectiva de las instituciones formadoras de maestros, no difiere significativamente entre docentes de los diferentes niveles educativos, sexo o edad.

La lectura es un proceso constructivo, pues en la medida de que vamos leyendo se activan nuestros esquemas y asumimos actitudes frente a ella. Por ello, es importante la técnica del diálogo, no solo con el texto, sino entre pares y con el profesor. De esa manera el texto cada vez nos dirá más, lo cual permitirá encontrarle un mejor sentido a los signos 
de puntuación, a los conectores y a los mecanismos de cohesión [4]

El texto es una manifestación, a través de los signos adecuados, de una idea, de un pensamiento, de una ilusión. Por consiguiente, es fundamental establecer la relación lógica de las ideas que se van tejiendo mediante los conectores pertinentes y. además, con los adverbios, pronombres, deíticos y otros mecanismos de cohesión. El saber emplearlos genera la familiaridad en el proceso lector.

Al incrementarse el acceso a la información en forma geométrica, el centro de la acción educativa pasó de las técnicas por retenerlas a las estrategias por procesarlas; es decir, desarrollar las potencialidades del ser humano para criticar y crear, darle valor agregado para construir conocimientos encaminados al desarrollo social. Por consiguiente, se impone la Pedagogía del Conocimiento, que no es otra que la pedagogía del procesamiento de la información; dicho de otra manera, de la comprensión y producción de textos, base de una educación científica y tecnológica. Es necesario reorientar hacia la Didáctica de la Comunicación [5].

Sabemos que tanto la comprensión como la producción tienen sus propios procesos, pero su interacción es evidente; por ejemplo, la identificación de la macroestructura textual implica una selección de ideas que las organizamos para elaborar un resumen, el cual es una expresión escrita. Por ello, consideramos que lograr el dominio de estrategias metacognitivas para una redacción eficaz no solo nos permitirá superar el gran problema que es plasmar por escrito nuestras ideas, sentimientos e intenciones, pues la escritura trasciende el simple trazado de símbolos convencionales para conformar signos, también nos permitirá mejorar en nuestra comprensión lectora, ya que lograremos una mayor conciencia de nuestras microhabilidades.
Es necesario elaborar materiales, en forma sistemática y de menor a mayor complejidad, así como lecturas que promuevan el acercamiento con las ciencias y las tecnologías, soporte de una educación que ayude al desarrollo sostenido. Esto servirá como aporte para los docentes de las instituciones educativas, los cuales deben ser enriquecidos en las aulas a través del monitoreo de las investigaciones de nuestra universidad

\section{Agradecimientos}

A la UNMSM que hace posible el desarrollo y difusión de nuestras investigaciones.

\section{Referencias}

\section{[1] ASOCIACIÓN DE ACADEMIAS} DE LA LENGUA ESPAÑOLA Y REAL ACADEMIA ESPAÑOL. Ortografía de la lengua española. Espasa Libros, 2010.

[2] CASSANY, D. Enseñar Lengua. Barcelona: Graó. (1998).

[3] GÓMEZ TORREGO, L. Hablar y escribir correctamente. Madrid, Editorial Arco. (2006).

[4] INGA. M. y CASAS, R. Módulo de Comprensión Lectora 6. Lima, Metrocolor. (2010).

[5] MARTíN, R. A. Manual de Didáctica de la Lengua y la Literatura. Madrid, Síntesis. (2009).

E-mail: miguelinga_unmsm@hotmail.com

projass2@yahoo.com

fvaras94@hotmail.com 